\title{
钯催化的烯丙基取代反应中烯丙基醇的高效活化
}

\author{
马献涛* 于 静王子龙张文武周秋菊 \\ (信阳师范学院化学化工学院 信阳市绿色催化和合成重点实验室 河南信阳 464000)
}

\begin{abstract}
摘要 由于烯丙基醇的碳一氧键较为惰性，烯丙基醇的高效活化是烯丙基取代反应研究中的一个重要方向. 近年来，非 经典的外加酸活化以及无外加活化剂条件下烯丙基醇的高效活化及取代反应得到了快速发展，为烯丙基醇的高效活化 提供了新的研究思路. 根据烯丙基醇的活化方式及机制对这些研究进展进行了简要论述.
\end{abstract}

关键词 烯丙基醇; 烯丙基取代; 钯催化; 酸或酸性氢活化; 氢键活化

\section{Efficient Activation of Allylic Alcohols in Pd-Catalyzed Allylic Substitution Reactions}

\author{
Ma, Xiantao* Yu, Jing Wang, Zilong Zhang, Yun Zhou, Qiuju \\ (Green Catalysis \& Synthesis Key Laboratory of Xinyang City, College of Chemistry and Chemical Engineering, \\ Xinyang Normal University, Xinyang, Henan 464000)
}

\begin{abstract}
Owing to the poor leaving character of hydroxy group, the efficient activation of allylic alcohols is important to allylic substitution reaction. In recent years, the efficient activation of allylic alcohols by non-typical acid activators and even no extra activators has been rapidly developed, and provides a novel approach to efficient activation of allylic alcohols. The recent developments in this area are summarized on the basis of different activation modes and mechanisms.

Keywords allylic alcohols; allylic substitution; Pd catalysis; activation by acid or acidic hydrogen; hydrogen bond activation
\end{abstract}

过渡金属催化的烯丙基取代反应，即在过渡金属催 化下烯丙基亲电试剂与亲核试剂发生取代反应生成一 分子的烯丙基化合物, 被广泛地应用于碳-碳、碳一杂化 学键的构建及天然产物的高效合成. 经过半个多世纪的 发展, 化学家们发现各种过渡金属如钯 ${ }^{[1-11]}$ 、铱 ${ }^{[12-16] 、}$ 镍 ${ }^{[17]} 、$ 铑 ${ }^{[18-19] 、}$ 、钓 ${ }^{[20]} 、$ 金 $^{[21]}$ 、铜 ${ }^{[22]}$ 等都可以催化烯丙基 取代反应. 其中, 以钯催化发展最为成熟, 研究最为深 入，应用最为广泛. 一般认为钯催化的烯丙基取代反应 的机理为: 首先零价钯催化剂和烯丙基亲电试剂进行烯 键配位, 再发生氧化加成生成关键的 $\pi$-烯丙基钯正离子 中间体，在此过程中，烯丙基亲电试剂的离去基团离去; 然后，亲核试剂进攻 $\pi$-烯丙基钯正离子中间体，得到烯 丙基产物, 同时钯催化剂解离再生并进入新的催化循环 (Scheme 1). 一般地, 烯丙基亲电试剂对钯催化剂氧化

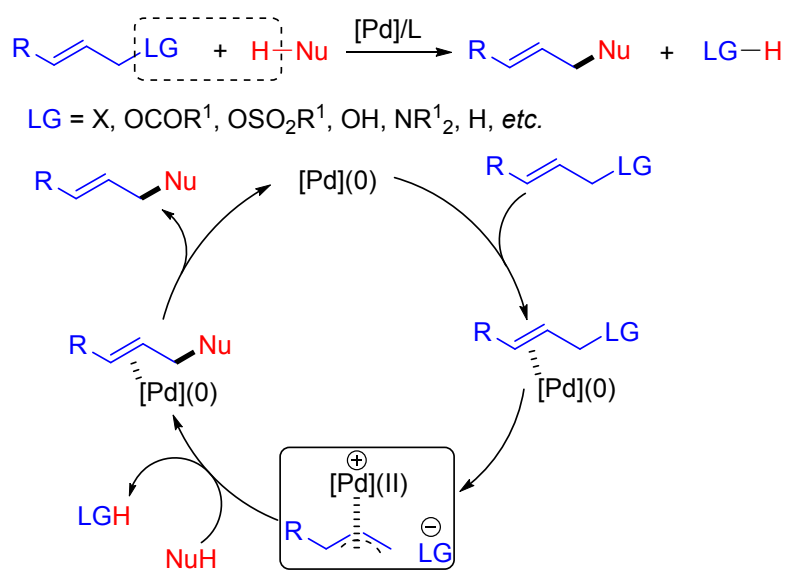

图式 1 钯催化的烯丙基取代反应的机理

Scheme 1 Reaction mechanism of Pd-catalyzed allylic substitution

\footnotetext{
* Corresponding author. E-mail: xiantaoma@126.com

Received May 6, 2020; revised May 24, 2020; published online June 11, 2020.

Project supported by the Key Scientific and Technological Project of Henan Province (No. 192102310031), the Key Scientific Research Project of Universities of Henan Province (No. 19B150018), the Nanhu Scholars Program for Young Scholars of Xinyang Normal University and the Young Core Instructor Program of Xinyang Normal University (No. 2018GGJS-05).

河南省科技攻关(No. 192102310031)、河南省高等学校重点科研(No. 19B150018)、信阳师范学院 “南湖学者奖励计划” 青年和信阳师范学院青年骨 干教师资助计划(No. 2018GGJS-05)资助项目.
} 
加成生成 $\pi$-烯丙基钯正离子中间体是整个反应的决速 步骤, 对反应起到至关重要的作用. 因此, 深入研究烯 丙基亲电试剂离去基团是如何离去的以及如何才能促 进氧化加成步骤的顺利发生一直是烯丙基取代反应研 究的重要内容.

烯丙基卤、烯丙基碳酸酯、烯丙基磺酸酯、烯丙基 磷酸酯、烯丙基醇、烯丙基胺、烯丙基碳氢化合物甚至 烯烃或炔烃等都被开发出来作为烯丙基亲电试剂[23-36]. 在众多的烯丙基亲电试剂中，烯丙基醇因具有廉价易 得、低毒稳定、参与反应时原子经济性好、副产物是水 等诸多优点, 得到了有机化学家们的广泛关注 ${ }^{[23-28]}$. 然 而, 由于烯丙基醇的碳-氧键比较惰性, 难以断裂, 传统 上一般需要将其衍生转化为烯丙基酯才能参与反应. 根 据烯丙基取代反应的机理， $\pi$-烯丙基钯正离子中间体的 有效生成是反应的关键步骤, 因此, 能否高效地实现烯 丙基醇碳一氧键的活化与断裂, 对于整个反应至关重 要 ${ }^{[23-28]}$. 近 20 年来, 烯丙基醇的高效活化研究一直是烯 丙基取代反应领域的一个热点.

由于酸可以与醇的氧原子结合, 极化醇的碳一氧键, 进而对醇的碳一氧键起到活化作用 ${ }^{[37]}$. 因此, 化学家们 最先就考虑到使用 Lewis 或 Brønsted 酸来实现对烯丙基 醇的活化. 大量的研究表明各种类型的 Lewis 酸、 Brønsted 酸 $\left[\right.$ 如 $\mathrm{B}(\mathrm{OH})_{3} 、 \mathrm{BEt}_{3} 、 \mathrm{As}_{2} \mathrm{O}_{3} 、 \mathrm{Ti}\left(\mathrm{O}-{ }^{i} \mathrm{Pr}\right)_{4} 、 \mathrm{PhCO}_{2} \mathrm{H}$ 、 $\mathrm{AcOH}$ 、手性磷酸、磷酸二苯酯等]均可以作为外加活化 剂, 用于钯催化的烯丙基醇碳一氧键的活化与断裂 (Scheme 2) ${ }^{[23-28]}$. 如 2004 年, Yamamoto 课题组 ${ }^{[38]}$ 采用 2 $\mathrm{mol} \% \mathrm{Pd}\left(\mathrm{PPh}_{3}\right)_{4}$ 和 $10 \mathrm{~mol} \%$ 醋酸体系可以催化烯丙基醇 与活泼亚甲基化合物的烯丙基取代反应. 该反应可以在 无溶剂的条件下, $100{ }^{\circ} \mathrm{C}$ 加热反应 $30 \mathrm{~min}$ 以高达 $98 \%$ 的 收率得到预期产物. 反应的底物可以是丙二酸酯类化合 物、 $\beta$-羰基酯类化合物以及 $\beta$-羰基砜类化合物等. 2014 年, 田仕凯课题组 ${ }^{[39]}$ 报道了钯催化下烯丙基醇与亚磺 酸钠的手性转移反应, 研究表明烯丙基醇在 4.0 equiv. $\mathrm{B}(\mathrm{OH})_{3}$ 的作用下才能高效地发生碳一氧键的断裂及手性 转移反应. 2014 年, 蒋高喜课题组 ${ }^{[40-41]}$ 报道了烯丙基醇 与二氢噁唑酮、吲哚酮等的烯丙基取代反应, 研究表明 催化量的苯甲酸对反应的顺利发生起到了至关重要的

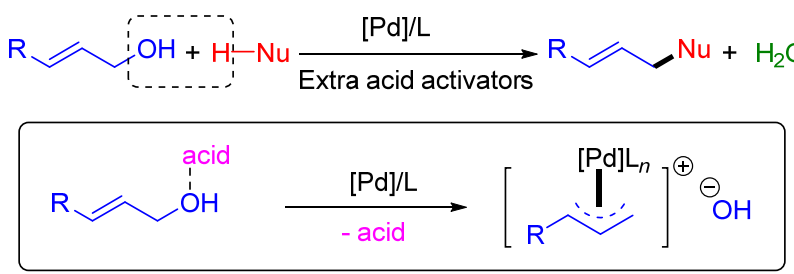

图式 2 外加酸性活化剂的烯丙基醇的活化与取代反应

Scheme 2 Activation and substitution of allylic alcohols under extra acid activators conditions
作用. 2019 年，曾晓飞、钟国富课题组 ${ }^{[42-43]}$ 报道了烯丙 基醇与 $\alpha$-杂环取代的酰胺、酯化合物的 $\alpha$-烯丙基取代反 应, 研究表明催化量的磷酸二苯酯可以促进烯丙基醇的 活化. 总之, 外加酸性活化剂仍然是当前实现烯丙基醇 的高效活化的主要方式之一.

与此同时，也有许多其他方式下烯丙基醇实现高效 活化, 参与烯丙基取代反应的报道, 如通过加入非经典 的酸性活化剂甚至无需外加活化剂条件下烯丙基醇的 活化. 本文拟结合课题组近年来在烯丙基醇的高效活化 及活化机制研究方面的经验，依据烯丙基醇的活化方式 及机制对该领域进行总结与综述，以期能够梳理烯丙基 醇的高效活化模型，为进一步设计、发展烯丙基醇的直 接取代反应提供思路与借鉴.

\section{1 非经典的酸性活化剂活化烯丙基醇的碳-氧 键}

除了上述的加入常见的 Lewis 酸、Brønsted 酸[如 $\mathrm{B}(\mathrm{OH})_{3} 、 \mathrm{BEt}_{3} 、 \mathrm{As}_{2} \mathrm{O}_{3} 、 \mathrm{Ti}\left(\mathrm{O}-{ }^{i} \mathrm{Pr}\right)_{4} 、 \mathrm{PhCO}_{2} \mathrm{H} 、 \mathrm{AcOH} 、$ 手性磷酸、磷酸二苯酯等]等活化剂实现烯丙基醇的活 化外，也有一些使用二氧化碳、磺酰肼等非经典的酸性 活化剂的报道.

\section{1 二氧化碳活化烯丙基醇的碳-氧键}

1996 年, Yamamoto 课题组 ${ }^{[44]}$ 发现在二氧化碳气体 的氛围下烯丙基醇的碳一氧键可以发生活化，与二乙基 胺或活泼亚甲基化合物发生烯丙基取代反应. 对照实验 显示钯催化剂及二氧化碳对反应缺一不可，这表明了二 氧化碳对烯丙基醇的活化作用. 机理研究表明烯丙基醇 与二氧化碳在钯催化下可以生成碳酸单烯丙酯, 由于羧 酸基团的拉电子效应, 烯丙基醇的碳一氧键被极化, 进 而易于断裂(Scheme 3).

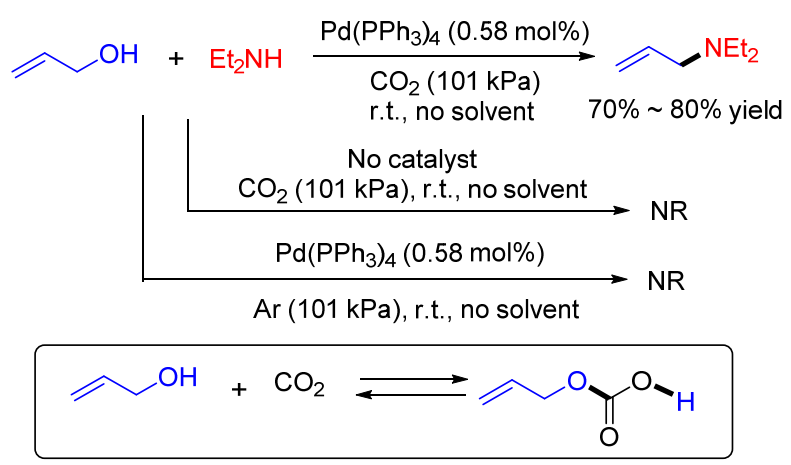

图式 3 二氧化碳对烯丙基醇的活化作用 Scheme 3 Activation of allylic alcohols by $\mathrm{CO}_{2}$

2014 年, Tunge 课题组 ${ }^{[45]}$ 报道了含有弱酸性氢的亲 核试剂如硝基化合物、腈、醛等与烯丙基醇的烯丙基取 代反应，用于制备 $\alpha$-位烯丙化的硝基化合物、腈、醛等 
(Eq. 1). 作者认为烯丙基醇与二氧化碳首先在钯催化下 生成碳酸单烯丙酯, 促进了氧化加成的过程, 进而利于 关键的 $\pi$-烯丙基钯正离子中间体的生成. 2017 年, Martin 课题组 ${ }^{[46]}$ 采用类似的二氧化碳活化烯丙基醇的策略, 实现了烯丙基醇向烯丙基羒酸的顺利转化.

$$
\begin{array}{ll}
\mathrm{EWG}=\mathrm{NO}_{2}, \mathrm{CN}, \mathrm{CHO} \\
\mathrm{DMSO}, 80^{\circ} \mathrm{C}
\end{array}
$$

2016 年, List 课题组 ${ }^{[4]}$ 报道了二氧化碳气体氛围下 取代环酮与烯丙基醇的直接取代反应, 实现了手性季碳 中心的高效构建, $e r$ 值高达 $95.5: 4.5$ (Eq. 2). 作者将新 方法应用于石蒜科生物碱 $(+)$-crinane 的对映选择性合成. 作者认为烯丙基醇可以在二氧化碳的作用下转化为高活 性的烯丙基碳酸酯, 促进了对钯催化剂氧化加成的过程, 进而利于关键的 $\pi$-烯丙基钯正离子中间体的生成.

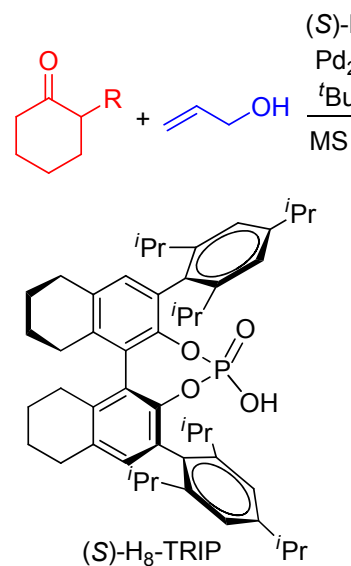

(S)- $\mathrm{H}_{8}$-TRIP (10 mol\%)

$\mathrm{Pd}_{2}(\mathrm{dba})_{3}(2.5 \mathrm{~mol} \%)$

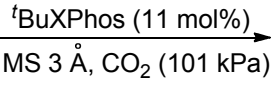

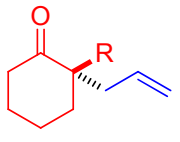

(S)- $\mathrm{H}_{8}$-TRIP $\quad{ }^{i} \mathrm{Pr}$

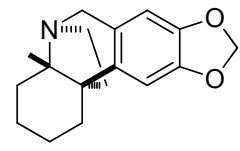

(+)-crinane
应的亚磺酸或磺酸，进而对烯丙基醇起到了活化作用. 同时，研究也表明单纯用亚磺酸或磺酸代替磺酰肼作为 活化剂时, 反应效果则较差, 这也体现了磺酰肼作为活 化剂具有一定的优势.

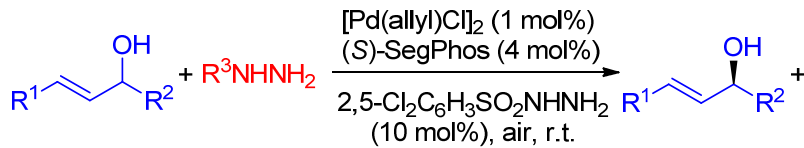

$$
\begin{aligned}
& s \text { factor up to }>400
\end{aligned}
$$

$\mathrm{R}^{1}=$ aryl, alkenyl, alkyl; $\mathrm{R}^{2}=$ aryl, alkyl

$\mathrm{R}^{3}=\mathrm{CO}_{2} \mathrm{R}, \mathrm{CONHR}, \mathrm{COR}, \mathrm{PO}(\mathrm{OR})_{2}$

\section{2 无外加活化剂下烯丙基醇的碳-氧键活化}

化学家们也一直致力于无外加活化剂下烯丙基醇 的活化研究. 已有报道表明配体、溶剂、反应底物和固 定相载体等都有可能对烯丙基醇起到活化作用.

\section{1 配体协同活化烯丙基醇的碳一氧键}

\section{1 .1 经历酸性氢物种}

2002 年, Ozawa 课题组 ${ }^{[52-53]}$ 合成了高活性的 [Pd]DPCB 催化剂, 首次实现了在无外加活化剂条件下烯丙 基醇与活泼亚甲基及胺类化合物的直接取代反应. 与传 统广为使用外加酸性活化剂的方法相比, 烯丙基醇在 $0.1 \mathrm{~mol} \%$ 钯催化剂及室温条件下即可高效地发生烯丙 基取代反应. 作者通过合成关键催化物种等方式对反应 的机理开展了研究, 结果表明反应中生成的酸性钯氢物 种促进了烯丙基醇碳一氧键的断裂(Scheme 4). 2006 年,

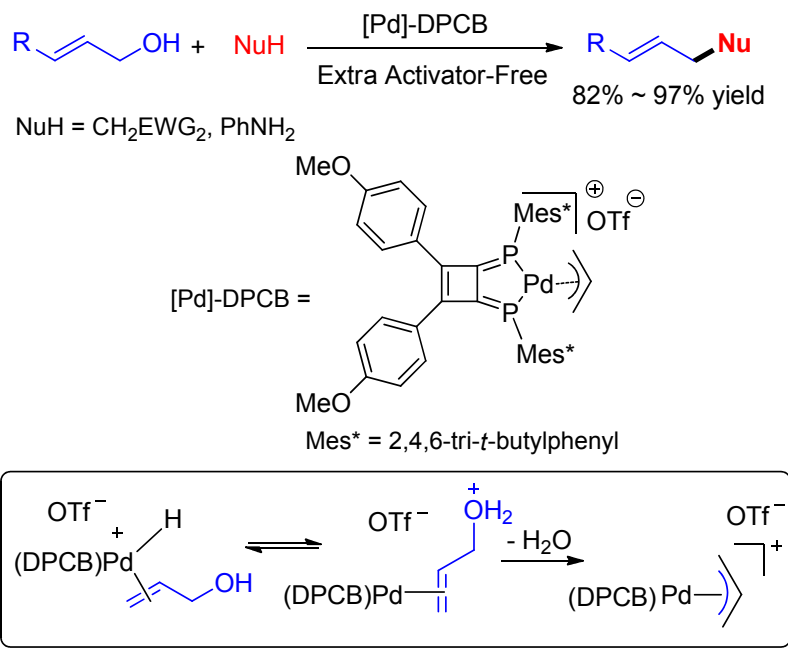

图式 4 经历酸性钯氢物种的烯丙基醇的活化与取代反应 Scheme 4 Activation and substitution of allylic alcohols via acidic $\mathrm{Pd}-\mathrm{H}$ species

丙基醇的动力学手性拆分, 选择性因子 $s$ 高达 $>400$ (Eq. 3). 研究表明磺酰胖在反应体系中有可能转化为相 
Le Floch 课题组 ${ }^{[54]}$ 通过理论计算对反应机理开展了研 究, 结果表明离子型的钯氢物种有可能首先发生质子转 移生成铵盐, 铵盐的氢质子对烯丙基醇的活化起到了重 要作用. 同时, 作者研究发现配体对整个反应过程至关 重要, 具有强 $\pi$-受体的配体(如亚磷酸三苯酯)对反应较 为有利.

2004 年, Ikariya 课题组 ${ }^{[5]}$ 报道了在无共催化剂、碱 添加剂的条件下, 烯丙基醇自身以及与醇亲核试剂的分 子间交叉烯丙基化反应(Eq. 4). 与其他广为使用的 $\mathrm{PPh}_{3} 、 1,2$-双 (二苯基膦)乙烷(DPPE)、1,4-双(二苯膦)丁 烷(DPPB)等富电子膦配体相比, 缺电子的 $\mathrm{P}(\mathrm{OPh})_{3}$ 表现 出明显的优势. 作者采用可分离的 $\mathrm{Pd}\left[\mathrm{P}(\mathrm{OPh})_{3}\right]_{4}$ 作为催 化剂, 结果表明 $\operatorname{Pd}\left[\mathrm{P}(\mathrm{OPh})_{3}\right]_{4}$ 是反应的活性催化物种, 在脱水烯丙基化反应过程中起到了关键的作用. 然后, 作者通过烯丙基醇与氛代甲醇的对照实验对反应机制 开展了研究, 结果表明烯丙基醇的自偶联以及与醇亲核 试剂的交叉偶联反应成醚的过程是可逆反应，会得到二 烯丙基醚以及烯丙基烷基醚的混合产物. 增大烯丙基醇 与醇亲核试剂的反应物配比, 可以促进交叉偶联反应的 发生，进而以较高的收率得到烯丙基烷基醚产物.

$$
\mathrm{CH}^{\mathrm{O} O H} \frac{\mathrm{Pd}\left[\mathrm{P}(\mathrm{OPh})_{3}\right]_{4}(0.5 \mathrm{~mol} \%)}{\mathrm{Ar}, 80^{\circ} \mathrm{C}} \mathrm{O}_{\mathrm{R}}+
$$

2011 年, Samec 课题组 ${ }^{[56]}$ 报道了 $\mathrm{Pd}\left[\mathrm{P}(\mathrm{OPh})_{3}\right]_{4}$ 催化 下芳香胺、磺酰胺与烯丙基醇的烯丙基取代反应. 在此 基础上，作者还发展了烯丙基取代-烯烃复分解的串联 反应，用于取代的二氢吡咯化合物的高效合成. 2014 年, Samec 课题组 ${ }^{[57]}$ 对该 $N$-烯丙基取代反应的机理开展了 深入的动力学、ESI-MS 以及立体化学研究. 结果表明反 应对烯丙基醇是二级动力学同位素效应，烯丙基醇的 氧一氢键与碳一氧键的断裂是反应的决速步骤. 反应机理 可能为: 首先烯丙基醇羟基的氧一氢键对钯催化剂氧化 加成, 生成了钯氢物种中间体; 在此钯氢物种作用下烯 丙基醇得到活化, 碳一氧键断裂生成关键的 $\pi$-烯丙基钯 正离子中间体; 最后, 由于 $\mathrm{P}(\mathrm{OPh})_{3}$ 强的 $\pi$-受体特性, 亲 核试剂从外围进攻 $\pi$-烯丙基钯正离子中间体, 进而得到 产物. 基于上述研究, 2018 年, Samec 课题组 ${ }^{[589]}$ 首次报 道了 Pd-BiPhePhos 催化下烯丙基醇与苯酚的烯丙基取 代反应, 可以单一选择性地得到 $O$-烯丙基化的产物. 研 究表明反应经历钯氢中间体活化烯丙基醇的过程, 与常 规的膦配体相比, $\pi$-酸性的双齿亚磷酸酯配体不易从钯 金属中心解离, 因此, 苯酚不易与钯金属配位, 进而避 免了 $C$-烯丙基取代反应的过程(Scheme 5). 得益于 BiPhePhos 的特殊性质, 同一年, Samec 课题组 ${ }^{[59]}$ 将
Pd-BiPhePhos 体系应用于烯丙基醇与 $N-, S$-, $C$-以及 $O$ 亲核试剂的手性转移反应, 手性转移效率高达 $>99 \%$ 。

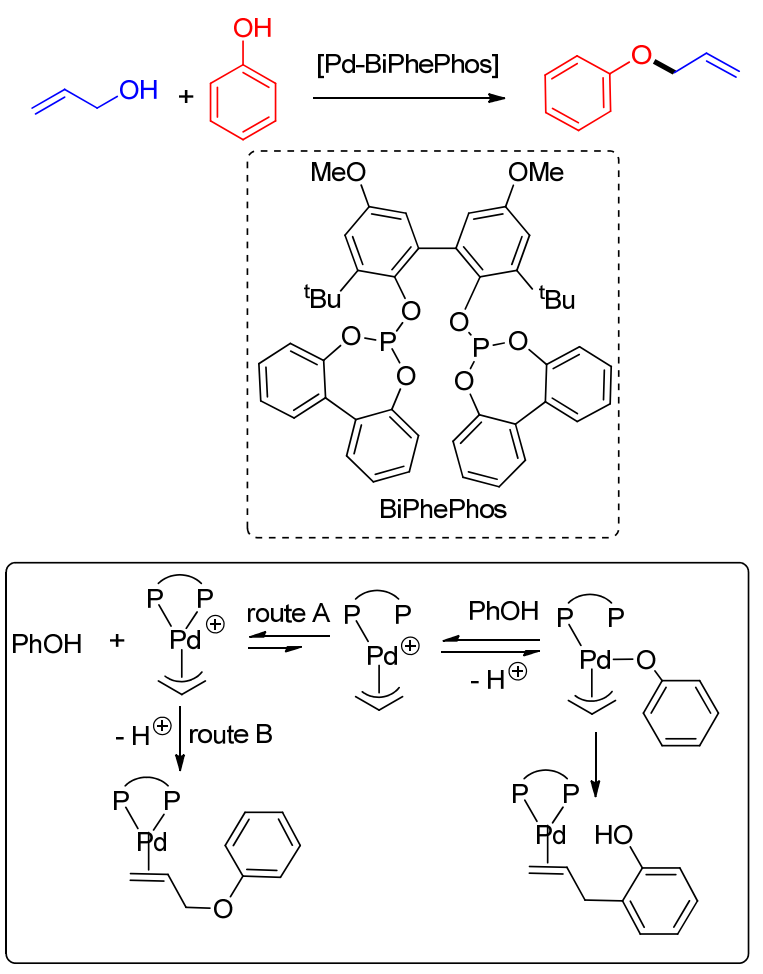

图式 5 苯酚与烯丙基醇的 $O$-烯丙基烷基化反应 Scheme $5 O$-Allylic alkylation of phenols with allylic alcohols

\subsection{2 通过氢键作用活化}

2008 年, Breit 课题组 ${ }^{[60]}$ 设计了 $\mathrm{Pd} / 6-\mathrm{DPPon}\left(\mathrm{CF}_{3}\right)_{2}$ 自 组装催化体系, 实现了 $N$-杂环化合物与烯丙基醇在无 外加活化剂条件下的直接取代反应，作者利用单晶衍射 及核磁检测技术研究证明反应可能经由氢键介导的环 簇体实现了对烯丙基醇的活化(Eq. 5).

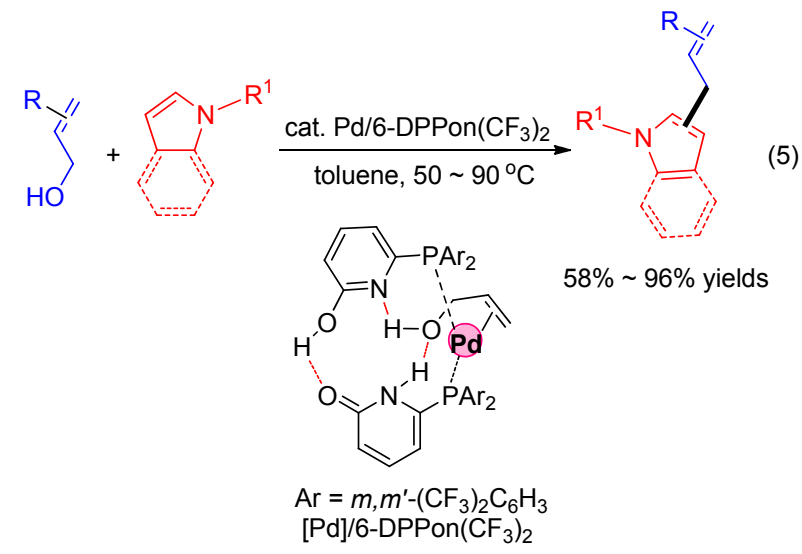

2014 年, Reek 课题组 ${ }^{[61-62]}$ 报道了钯催化下烯丙基醇 与吲哚或胺类化合物的烯丙基取代反应. 动力学研究显 示烯丙基醇、脲添加剂和钯催化剂表现出一级动力学效 应，亲核试剂表现出零级动力学效应，这表明烯丙基醇 
对钯催化剂的氧化加成过程是整个反应的决速步骤, 并 且嫝添加剂可以促进该氧化加成过程; 进一步的理论计 算研究表明嫝添加剂和配体通过氢键作用协同实现了 对烯丙基醇的活化(Scheme 6). 根据这些实验事实, 作 者提出了如下的反应机理: 首先 $\left[\left(\eta^{3}\right.\right.$-allyl $\left.) \mathrm{Pd}(\operatorname{cod})\right] \mathrm{BF}_{4}$ 与配体作用生成活性催化剂前体 $\mathbf{A}$, 然后亲核试剂进攻 $\mathbf{A}$ 得到中间体 $\mathbf{B}$, 烯丙基醇与中间体 $\mathbf{B}$ 中心的钯配位得 到中间体 $\mathbf{C}$, 同时释放出预期产物. 中间体 $\mathbf{C}$ 在腿添加 剂、配体的协同氢键作用经由 $\mathbf{D}$ 实现了对烯丙基醇的活 化, 再生了活性催化剂前体 $\mathbf{A}$, 并进入下一个催化循环.
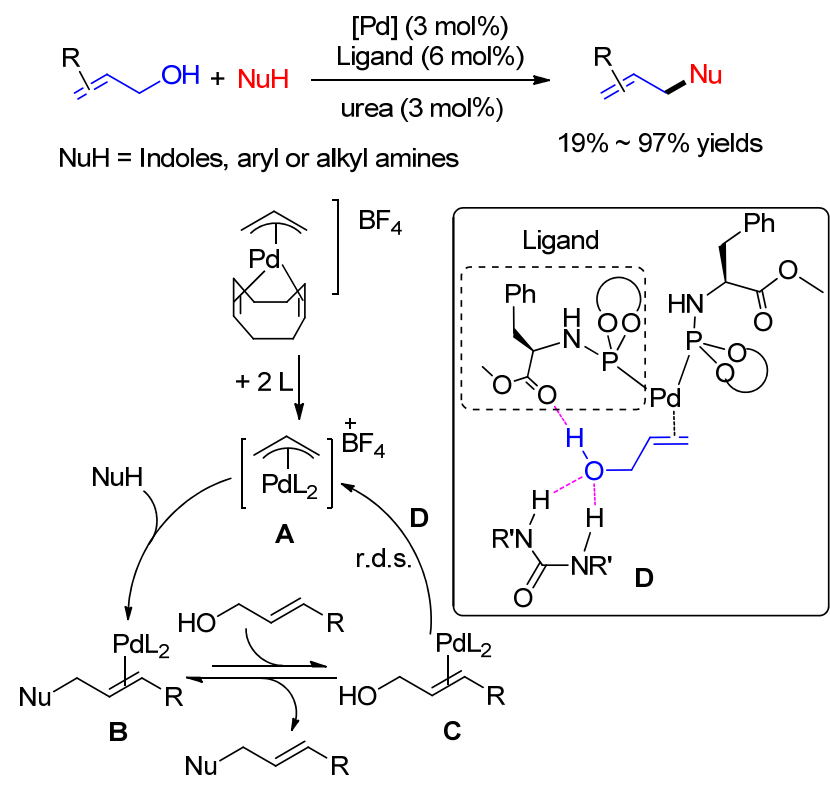

图式 6 腿添加剂、配体通过氢键作用协同实现了对烯丙基醇 的活化

Scheme 6 Activation of allylic alcohols via H-bonding of urea and ligand

\subsection{3 通过 Lewis 酸作用活化}

已有大量文献报道表明 Lewis 酸可以活化醇的碳氧键. 2006 年, Baba 课题组 ${ }^{[63]}$ 报道了 $\mathrm{InCl}_{3}$ 催化下活泼烯 丙基醇与吲哚、活泼亚甲基化合物等的烯丙基取代反应; 2007 年, 周锡庚课题组 ${ }^{[64]}$ 报道了 $\mathrm{Yb}(\mathrm{OTf})_{3}$ 催化下活泼 烯丙基醇与 1,3-二羰基化合物的烯丙基取代反应. 此外, Lewis 酸也作为外加活化剂被广泛地应用于钯催化下烯 丙基醇的烯丙基取代反应中 ${ }^{[25-28]}$. 受此启发, 2017 年, Kimura 课题组 ${ }^{[65]}$ 设计了膦-硼配体, 其可以在室温下实 现烯丙基醇与胺类化合物的烯丙基取代反应. 作者认为 膦中心起到了稳定钯催化剂的作用, 嗍中心可以作为 Lewis 酸位点活化烯丙基醇的羟基, 以促进其离去并生 成关键的 $\pi$-烯丙基钯正离子中间体(Scheme 7). 2018 年, 同一课题组 ${ }^{[66]}$ 又采用类似的膦-硼催化体系实现了烯丙 基醇与活泼亚甲基化合物的烯丙基取代反应.

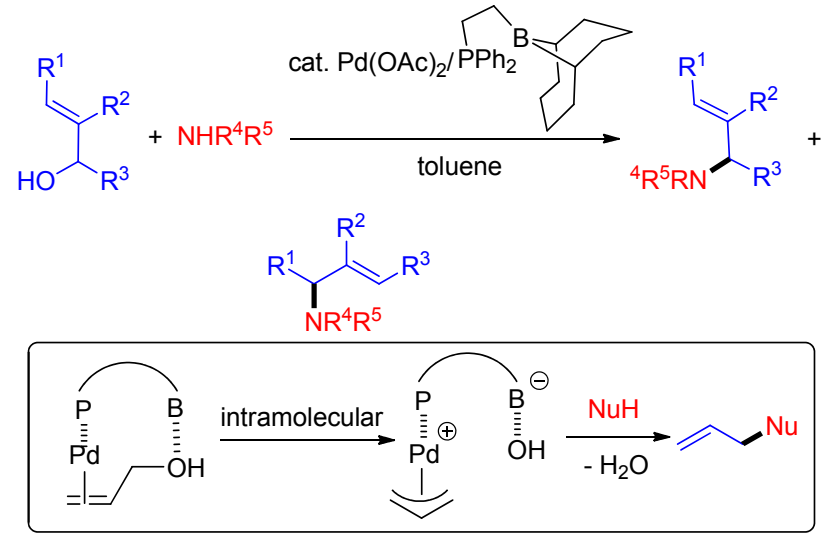

图式 7 P-B 配体介导的胺的 $N$-烯丙基烷基化反应 Scheme $7 N$-Allylic alkylation of amines mediated by a P-B ligand

\section{2 溶剂协同活化烯丙基醇的碳-氧键}

\subsection{1 水溶剂协同活化烯丙基醇的碳一氧键}

2004 年, Oshima 课题组 ${ }^{[67]}$ 报道了首例水氢键活化 下烯丙基醇与活泼亚甲基化合物或胺的取代反应. 作者 通过理论计算对水的作用进行了研究, 结果表明水可以 通过水合作用实现对烯丙基醇的活化，并且水对离去基 团氢氧根起到一定的稳定作用, 进而极大地降低了反应 的活化能, 利于反应在温和条件下顺利地发生(Scheme 8). 2011 年, Hikawa 与 Yokoyama 课题组 ${ }^{[68]}$ 报道了水介质 中氨基酸与烯丙基醇的 $N$-烯丙基取代反应，作者认为 水通过水合作用，在烯丙基醇的活化及 $\pi$-烯丙基钯正离 子中间体的生成过程中起到了重要的作用. 2012 年, Yang 课题组 ${ }^{[69]}$ 报道了水相体系中烯丙基醇与活泼亚甲 基化合物的烯丙基取代反应. 2015 年, Kato 课题组 ${ }^{[70]}$ 将 烯丙基醇与内炔化合物的串联环化反应用于吲哚、苯并 呋喃、苯并噻吩化合物的合成. 这些作者均提出了水对 烯丙基醇的水合活化作用.

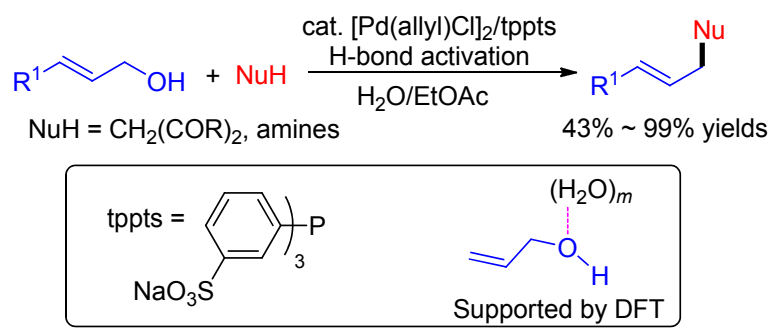

图式 8 基于理论计算的水氢键活化烯丙基醇研究 Scheme 8 Studies on the activation of allyl alcohols by $\mathrm{H}$-bonds of water based on theoretical calculations

2019 年, 马献涛课题组 ${ }^{[71]}$ 报道了水相体系中烯丙 基醇与稳定磷叶立德的脱水取代反应. 为了获取更为直 观的关于氢键的实验性证据，作者 ${ }^{[72-73]}$ 采用扩散序谱 
(DOSY)的方式对水与烯丙基醇之间的作用开展了研究 (Scheme 9). 结果表明烯丙基醇与水之间通过氢键生成 了扩散半径更大的物种, 所以导致水的扩散系数发生了 明显的下降. 基于这样的实验事实, 作者认为水通过氢 键实现了对烯丙基醇的活化.

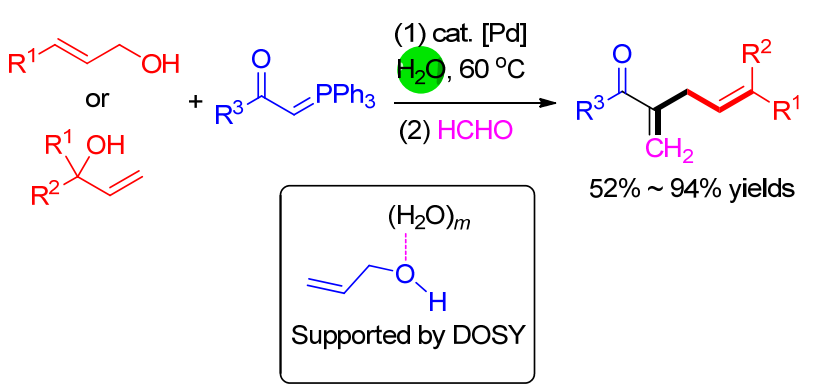

图式 9 基于 DOSY 的水氢键活化烯丙基醇研究

Scheme 9 Studies on the activation of allylic alcohols by $\mathrm{H}$-bonds of water based on DOSY

\subsection{2 醇溶剂协同活化烯丙基醇的碳一氧键}

2014 年, 张万斌课题组 ${ }^{[74]}$ 报道了钯催化下, 醛、酮 在吡咯烷的活化下与烯丙醇在室温条件下的直接取代 反应, 实现了醛酮 $\alpha$-位的烯丙基官能化. 作者通过理论 计算对甲醇的作用开展了研究, 认为甲醇通过氢键实现 了对烯丙基醇的活化, 并且甲醇参与了反应过渡态的形 成, 可以极大地降低反应的活化能, 因此, 该反应可以 在室温下发生(Scheme 10). 随后, 张万斌课题组 ${ }^{[75]}$ 将溶 剂氢键活化的理念应用于烯丙基醇与胺在室温条件下 的直接取代反应.

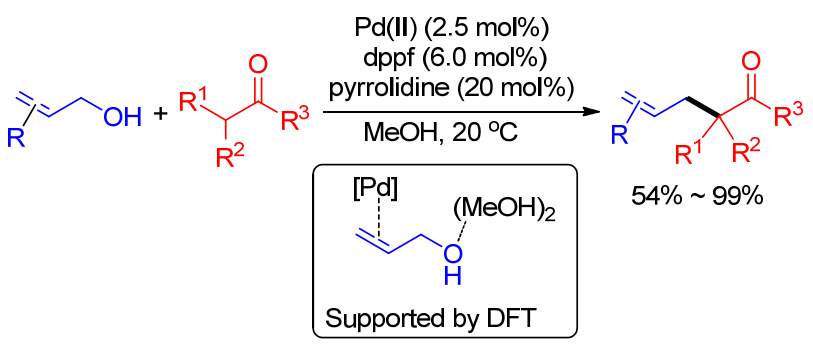

图式 10 基于理论计算的甲醇氢键活化烯丙基醇研究

Scheme 10 Studies on the activation of allylic alcohols by $\mathrm{H}$-bonds of $\mathrm{MeOH}$ based on theoretical calculations

\section{3 底物通过协同氢键作用活化烯丙基醇的碳一氧键}

\subsection{1 底物中含有羧酸基团}

2011 年, Hikawa 与 Yokoyama 课题组 ${ }^{[76]}$ 报道了水相 体系中 2-氨基苯甲酸与烯丙基醇的选择性 $N$-烯丙基取 代反应(Eq. 6). 作者认为反应底物中的羧酸基团可以起 到质子酸的作用实现对烯丙基醇的活化. 水在羧酸的离 子化过程以及质子从羧酸基团向醇羟基的转移过程起 到了重要的作用.

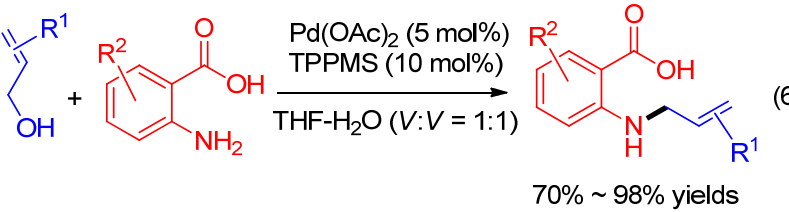

2018 年, Kleij 课题组 ${ }^{[77]}$ 报道了钯催化下 $\alpha$-差等基羧 酸与胺的烯丙化一环化反应，用于 $\alpha, \beta$-不饱和 $\gamma$-内酰胺 化合物的高效合成. 研究表明羧酸基团与羟基之间的氢 键作用对烯丙基醇的活化并没有起到重要的作用. 各种 光谱学及对照实验表明羧酸的氧一氢键首先对钯催化剂 氧化加成, 生成钯氢物种, 然后发生氢转移实现对烯丙 基醇的活化，进而生成关键的 $\pi$-烯丙基钯正离子中间 体; 最后，羧酸基的氧亲核位点进攻钯金属中心，还原 消除得到预期产物并再生钯催化剂(Scheme 11). 随后, Kleij 课题组 ${ }^{[78]}$ 报道了类似的 $\alpha$-差基羧酸与二氢吲哚的 $N$-烯丙基取代反应，用于 $\alpha, \beta$-不饱和 $\gamma$-氨基酸化合物的 高效合成.

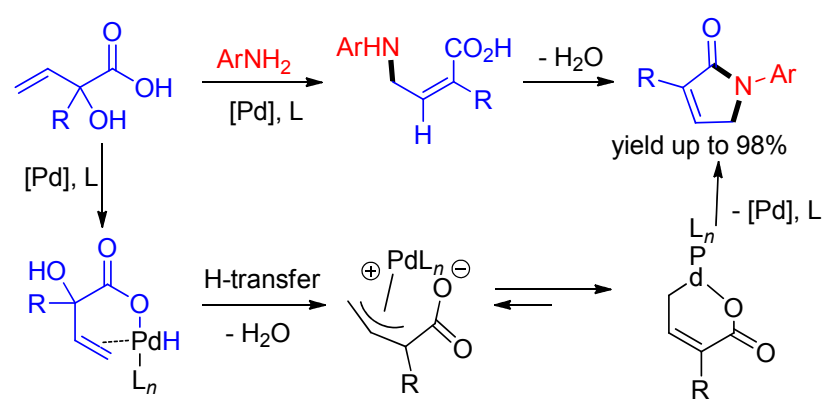

图式 11 不饱和内酰胺的多米诺合成

Scheme 11 Domino synthesis of unsaturated lactams

2019 年, Ohmiya 课题组 ${ }^{[79]}$ 报道了 $\mathrm{Pd}(\mathrm{TFA})_{2} / 1,1^{\prime}$-双 (二异丙基膦)二茂铁(DIPPF)与卡宾催化剂协同催化下 醛与烯丙基醇的烯丙基取代反应，用于烯丙基酮类化合 物的合成. 理论计算表明卡宾催化剂与醛形成的烯醇式 中间体有可能作为 Brønsted 酸与烯丙基醇的羟基结合, 进而实现对烯丙基醇的活化(Scheme 12).

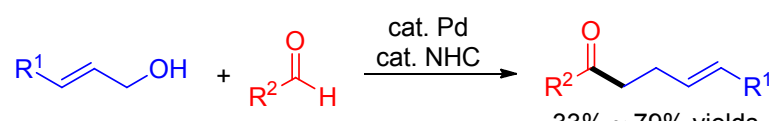
$33 \% \sim 79 \%$ yields

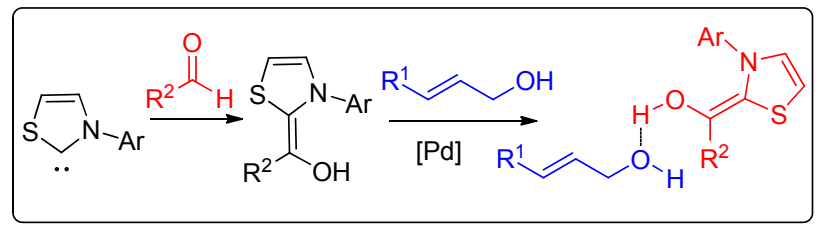

图式 12 醛与烯丙基醇的脱水烯丙基烷基化反应

Scheme 12 Dehydrative allylation between aldehydes and allylic alcohols 


\subsection{2 底物通过氢键作用活化}

2014 年, 蒋高喜课题组 ${ }^{[80]}$ 报道了烯丙基醇与二氢 啞唑酮的烯丙基取代反应. 作者推测二氢噁唑酮可以发 生烯醇异构进而可以与烯丙基醇以氢键方式形成六元 环簇, 实现对烯丙基醇的活化. 此外, 文献报道表明烯 丙基醇与底物之间的氢键作用是普遍存在的 (Scheme 13). 2017 年, 解沛忠、罗德平课题组 ${ }^{[81]}$ 报道了 MoritaBaylis-Hillma (MBH) 类型烯丙基醇与二级氧磷化合物 的烯丙基取代反应. 作者通过理论计算研究表明二级膦 氧化合物可以发生异构转化为二取代的亚磷酸, 然后通 过氢键实现对烯丙基醇的活化作用. 随后，作者 ${ }^{[82]}$ 将该 理念应用于 $\mathrm{MBH}$ 类型烯丙基醇与亚磺酸化合物的烯丙 基取代反应. 同样地, 作者通过理论计算证明了亚磺酸 通过氢键对烯丙基醇的活化作用.
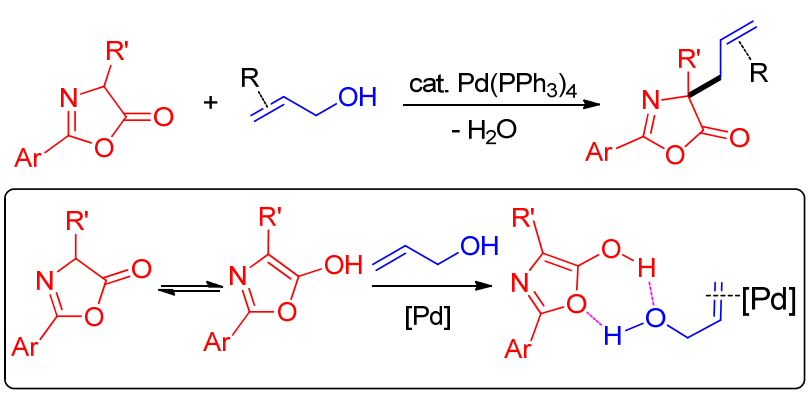

图式 13 六元环簇活化烯丙基醇的设想

Scheme 13 Hypothesis on activation of allylic alcohols via an six-member ring species

2019 年, 马献涛课题组 ${ }^{[83-84]}$ 在研究烯丙基醇与稳 定磷叶立德的脱水取代反应时意外地发现在非极性、非 质子溶剂如环已烷、甲苯等溶剂中, 反应仍可以发生. 这与之前该课题报道的利用水介质的氢键作用活化烯 丙基醇的作用机制不符 ${ }^{[71]}$. 受蒋高喜课题组 ${ }^{[80]}$ 研究报 道的启发, 作者推测反应体系中有可能存在类似的六元 环簇结构, 进而实现了对烯丙基醇的活化. 为此, 作者 借助于变温核磁、DOSY、Job Plot、NMR 滴定、二维 NOESY 等核磁技术对烯丙基醇的活化机制开展了研

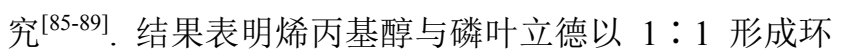
状复合物种(Scheme 14). 根据这些实验事实, 作者认 为, 在水相体系中, 水通过氢键实现对烯丙基醇的活化 是主要的反应路径; 而在非极性、非质子溶剂中, 磷叶 立德通过氢键与膦氧非共价键的方式与烯丙基醇形成 六元环簇结构，进而实现了对烯丙基醇的活化.

基于以上文献报道及研究经验, 马献涛课题组认为 具有氢键供体与受体位点的亲核试剂底物与烯丙基醇 形成六元环族物种有可能是实现烯丙基醇活化的一种 有效方式. 异构化的杂芳环化合物, 如 2-羟基吡啶存在 烯醇式与酮式结构的异构现象 ${ }^{[90-92]}$, 既可以作为氢键的<smiles>[R]C/C=C\CO[Pb]1=CC([R])=CO1</smiles>

- Job Plot and ${ }^{1} \mathrm{H}$ NMR Titration $\circ 2 \mathrm{D}$ NOESY

- Variable-Temperature ${ }^{1} \mathrm{H}$ NMR $\circ$ DOSY

图式 14 底物辅助的次级键活化烯丙基醇的实验证据 Scheme 14 Experimental evidences on the substrate selfassisted secondary bond activation of allylic alcohols

给体也可以作为氢键的供体, 然而, Cook 课题组 ${ }^{[93]}$ 在研 究异构化杂芳环化合物与烯丙基醇的直接取代反应中 并未重视到这一现象. 马献涛课题组分析 Cook 课题组 的研究数据发现异构化杂芳环化合物与烯丙基醇的烯 丙基取代反应可以在甲苯、苯等非极性、非质子溶剂中 顺利发生，这说明极有可能存在底物通过六元环簇物种 实现对烯丙基醇的活化过程. 为证实该猜想, 2020 年, 马献涛课题组 ${ }^{[94]}$ 利用变温核磁、Job Plot、NMR 滴定等 实验技术对肉桂醇与 2-羟基吡啶之间的相互作用开展 研究, 结果表明二者之间可以通过氢键作用形成 $1: 1$ 及 $1: 2$ 类型的复合物种; 通过对照实验进一步证明 1 : 1 的环簇复合物种在烯丙基醇的高效活化中起到至关重 要的作用(Scheme 15). 在此指导下, 作者发展了温和条 件下基于底物氢键活化的异构化杂芳环的烯丙基取代 反应新方法.

$$
\text { Supported by Job plot and }{ }^{1} \mathrm{H} \text { NMR Titration }
$$

图式 15 异构化杂芳环与烯丙基醇的烯丙基取代反应 Scheme 15 Allylic substitution of tautomerizable heteroarenes with allylic alcohols

\section{4 固载载体辅助活化烯丙基醇的碳-氧键}

2018 年, Motokura 课题组 ${ }^{[95]}$ 报道了硅胶固载的钯催 化剂作用下烯丙基醇与活泼亚甲基化合物的烯丙基取 代反应, 转化数(TON) 高达 4500 (Scheme 16). 作者认为 硅胶中裸露的羟基对烯丙基醇的活化起到了重要的作 用. 2020 年, Motokura 课题组 ${ }^{[96]}$ 又进一步对硅胶的孔径、 碱添加剂等影响因素进行了研究.

\section{3 结论与展望}

综上所述，烯丙基醇的高效活化是烯丙基取代反应 研究中的一个重要课题. 烯丙基醇的活化机制的研究有 助于促进烯丙基醇高效活化新方法的开发及建立. 目前 


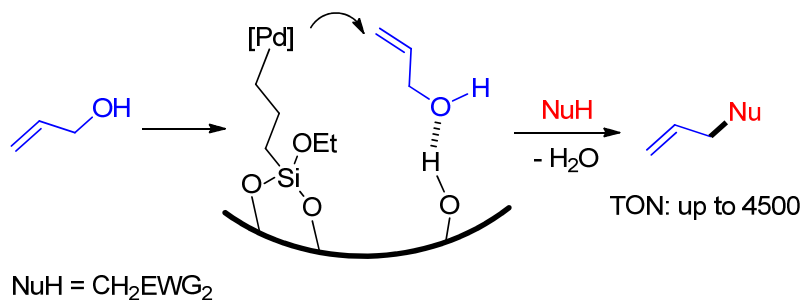

图式 16 硅胶固载载体促进的烯丙基取代反应

Scheme 16 Silica support-enhanced allylic substitution reactions

的研究报道中烯丙基醇的活化方式可以大致分为外加 活化剂活化、配体辅助活化、溶剂氢键协同活化、底物 辅助活化、固载载体辅助活化等几种，从活化机制的本 质上来讲可以分为酸或酸性氢活化、氢键活化两类. 其 中，基于酸或酸性氢活化的反应机制，化学家们已从实 验及理论计算的层面开展了较为深入的研究, 极大地促 进了该活化策略的快速发展. 但是, 由于氢键的特殊性 质，目前基于氢键活化的机制研究仍主要借助于理论计 算的手段, 仍严重贵乏实验性的证据, 因此, 发展合理 有效的研究氢键活化的实验方法仍是该领域亟待解决 的重要问题. 另外, 基于无外加活化剂理念的烯丙基醇 的活化及取代反应研究虽有一些不对称合成的报道, 但 仍有待进一步地深化与发展.

\section{References}

[1] Tsuji, J. Acc. Chem. Res. 1969, 2, 144

[2] Trost, B. M. Tetrahedron 1977, 33, 2615.

[3] Trost, B. M.; Van Vranken, D. L. Chem. Rev. 1996, 96, 395.

[4] Trost, B. M.; Crawley, M. L. Chem. Rev. 2003, 103, 2921.

[5] Dai, L.-X.; Tu, T.; You, S.-L.; Deng, W.-P.; Hou, X.-L. Acc. Chem. Res. 2003, 36, 659.

[6] Trost, B. M.; Machacek, M. R.; Aponick, A. Acc. Chem. Res. 2006, 39,747

[7] Lu, Z.; Ma, S. Angew. Chem., Int. Ed. 2008, 47, 258.

[8] Trost, B. M. Org. Process Res. Dev. 2012, 16, 185.

[9] Chen, D.-F.; Han, Z.-Y.; Zhou, X.-L.; Gong, L.-Z Acc. Chem. Res. 2014, 47, 2365.

[10] Zhang, M.-M.; Luo, Y.-Y.; Lu, L.-Q.; Xiao, W.-J. Acta Chim. Sinica 2018, 76, 838 (in Chinese). (张毛毛，骆元元，陆良秋，肖文精，化学学报，2018，76, 838.)

[11] Zhang, H.-H.; Yu, S. Acta Chim. Sinica 2019, 77, 832 (in Chinese). (张洪浩, 俞寿云, 化学学报, 2019, 77, 832.)

[12] Hartwig, J. F.; Stanley, L. M. Acc. Chem. Res. 2010, 43, 1461.

[13] Zhuo, C.-X.; Zheng, C.; You, S.-L. Acc. Chem. Res. 2014, 47, 2558.

[14] Deng, Y.; Yang, W.; Yang, X.; Yang, D. Chin. J. Org. Chem. 2017, 37, 3039 (in Chinese) (邓颖颖，杨文，杨新，杨定乔，有机化学，2017，37, 3039.)

[15] Cheng, Q.; Tu, H.-F.; Zheng, C.; Qu, J.-P.; Helmchen, G.; You, S.-L. Chem. Rev. 2019, 119, 1855

[16] Shen, D.; Chen, Q.; Yan, P.; Zeng, X.; Zhong, G. Angew. Chem., Int. Ed. 2017, 56, 3242.

[17] Zhang, H.; Gu, Q.; You, S. Chin. J. Org. Chem. 2019, 39, 15 (in Chinese).

(张慧君，顾庆，游书力，有机化学， 2019, 39, 15.)

[18] Turnbull, B. W. H.; Evans, P. A. J. Org. Chem. 2018, 83, 11463.

[19] Thoke, M. B.; Kang, Q. Synthesis 2019, 51, 2585.

[20] Bruneau, C.; Renaud, J.-L.; Demerseman, B. Chem.-Eur. J. 2006,
12,5178 .

[21] Quintavalla, A.; Bandini, M. ChemCatChem 2016, 8, 1437.

[22] Alexakis, A.; Bäckvall, J. E.; Krause, N.; Pàmies, O.; Diéguez, M. Chem. Rev. 2008, 108, 2796.

[23] Muzart, J. Tetrahedron 2005, 61, 4179

[24] Bandini, M. Angew. Chem., Int. Ed. 2011, 50, 994.

[25] Sundararaju, B.; Achard, M.; Bruneau, C. Chem. Soc. Rev. 2012, $41,4467$.

[26] Bandini, M.; Cera, G.; Chiarucci, M. Synthesis 2012, 44, 504.

[27] Butta, N. A.; Zhang, W. Chem. Soc. Rev. 2015, 44, 7929.

[28] Qian, J.; Jiang, G. Curr. Catal. 2017, 6, 25.

[29] Tang, H.; Huo, X.; Meng, Q.; Zhang, W. Acta Chim. Sinica 2016 74,219 (in Chinese). (汤添溟, 霍小红, 孟庆华, 张万斌, 化学学报, 2016, 74, 219.)

[30] Wang, P.-S.; Shen, M.-L.; Gong, L.-Z. Synthesis 2018, 50, 956.

[31] Li, G.; Huo, X.; Jiang, X.; Zhang, W. Chem. Soc. Rev. 2020, 49, 2060.

[32] Gao, S.; Liu, H.; Wu, Z.; Yao, H.; Lin, A. Green Chem. 2017, 19, 1861.

[33] Zheng, J.; Breit, B. Org. Lett. 2018, 20, 1866.

[34] Lu, C.; Yu, X.; Chen, D.; Wang, H.; Song, Q.; Gao, J. Org. Biomol. Chem. 2019, 17, 3545.

[35] Ren, W.; Zuo, Q.-M.; Niu, Y.-N.; Yang, S.-D. Org. Lett. 2019, 21 , 2596.

[36] Lu, C.-J.; Yu, X.; Chen, Y.-T.; Song, Q.-B.; Yang, Z.-P.; Wang, H. Eur. J. Org. Chem. 2020, 2020, 680.

[37] Chen, L.; Yin, X.-P.; Wang, C.-H.; Zhou, J. Org. Biomol. Chem. 2014, 12, 6033.

[38] Patil, N. T.; Yamamoto, Y. Tetrahedron Lett. 2004, 45, 3101

[39] Ma, X.-T.; Dai, R.-H.; Zhang, J.; Gu, Y.; Tian, S.-K. Adv. Synth. Catal. 2014, 356, 2984.

[40] Zhou, H.; Yang, H.; Liu, M.; Xia, C.; Jiang, G. Org. Lett. 2014, 16 , 5350 .

[41] Yang, H.; Zhou, H.; Yin, H.; Xia, C.; Jiang, G. Synlett 2014, 25, 2149

[42] Pan, S.; Wu, B.; Hu, J.; Xu, R.; Jiang, M.; Zeng, X.; Zhong, G. $J$. Org. Chem. 2019, 84, 10111

[43] Yan, P.; Pan, S.; Hu, J.; Lu, L.; Zeng, X.; Zhong, G. Adv. Synth. Catal. 2019, 361, 1322.

[44] Sakamoto, M.; Shimizu, I.; Yamamoto, A. Bull. Chem. Soc. Jpn. 1996, 69, 1065.

[45] Lang, S. B.; Locascio, T. M.; Tunge, J. A. Org. Lett. 2014, 16, 4038.

[46] van Gemmeren, M.; Bçrjesson, M.; Tortajada, A.; Sun, S.-Z.; Okura, K.; Martin, R. Angew. Chem., Int. Ed. 2017, 56, 6558.

[47] Pupo, G.; Properzi, R.; List, B. Angew. Chem., Int. Ed. 2016, 55, 6099.

[48] Fu, M.-C.; Shang, R.; Cheng, W.-M.; Fu, Y. Chem.-Eur. J. 2017, 23, 8818.

[49] Wu, F.-P.; Peng, J.-B.; Fu, L.-Y.; Qi, X.; Wu, X.-F. Org. Lett. 2017, 19, 5474.

[50] Li, M.-B.; Li, H.; Wang, J. Liu, C.-R.; Tian, S.-K. Chem. Commun. 2013, 49, 8190.

[51] Yan, L.; Xu, J.-K.; Huang, C.-F.; He, Z.-Y.; Xu, Y.-N.; Tian, S.-K. Chem.-Eur. J. 2016, 22, 13041

[52] Ozawa, F.; Okamoto, H.; Kawagishi, S.; Yamamoto, S.; Minami, T.; Yoshifuji, M. J. Am. Chem. Soc. 2002, 124, 10968.

[53] Ozawa, F.; Ishiyama, T.; Yamamoto, S.; Kawagishi, S.; Murakami, H. Organometallics 2004, 23, 1698.

[54] Piechaczyk, O.; Thoumazet, C.; Jean, Y.; le Floch, P. J. Am. Chem. Soc. 2006, 128, 14306

[55] Kayaki, Y.; Koda, T.; Ikariya, T. J. Org. Chem. 2004, 69, 2595.

[56] Sawadjoon, S.; Samec, J. S. M. Org. Biomol. Chem. 2011, 9, 2548.

[57] Sawadjoon, S.; Sjçberg, P. J. R.; Orthaber, A.; Matsson, O.; Samec, J. S. M. Chem.-Eur. J. 2014, 20, 1520 .

[58] Rukkijakan, T.; Akkarasamiyo, S.; Sawadjoon, S.; Samec, J. S. M. J. Org. Chem. 2018, 83, 4099.

[59] Akkarasamiyo, S.; Sawadjoon, S.; Orthaber, A.; Samec, J. S. M. 
Chem.-Eur. J. 2018, 24, 3488.

[60] Usui, I.; Schmidt, S.; Keller, M.; Breit, B. Org. Lett. 2008, 10, 1207.

[61] Gumrukcu, Y.; de Bruin, B.; Reek, J. N. H. ChemSusChem 2014, 7, 890.

[62] Gumrukcu, Y.; de Bruin, B.; Reek, J. N. H. Catalysts 2015, 5, 349.

[63] Yasuda, M.; Somyo, T.; Baba, A. Angew. Chem., Int. Ed. 2006, 45, 793.

[64] Huang, W.; Wang, J.; Shen, Q.; Zhou, X. Tetrahedron Lett. 2007, 48, 3969.

[65] Hirata, G.; Satomura, H.; Kumagae, H.; Shimizu, A.; Onodera, G.; Kimura, M. Org. Lett. 2017, 19, 6148.

[66] Shimizu, A.; Hirata, G.; Onodera, G.; Kimura, M. Adv. Synth. Catal. 2018, 360, 1954.

[67] Kinoshita, H.; Shinokubo, H.; Oshima, K. Org. Lett. 2004, 6, 4085.

[68] Hikawa, H.; Yokoyama, Y. Org. Biomol. Chem. 2011, 9, 4044.

[69] Shue, Y.-J.; Yang, S.-C. Tetrahedron Lett. 2012, 53, 1380.

[70] Dhage, Y. D.; Shirai, T.; Arima, M.; Nakazima, A.; Hikawa, H.; Azumaya, I.; Kusakabe, T.; Takahashi, K.; Kato, K. RSC Adv. 2015, $5,42623$.

[71] Ma, X.; Yu, J.; Han, C.; Zhou, Q.; Ren, M.; Li, L.; Tang, L. Adv. Synth. Catal. 2019, 361, 1023.

[72] Johnson, C. S. Prog. Nucl. Magn. Reson. Spectrosc. 1999, 34, 203.

[73] Cohen, Y.; Avram, L.; Frish, L. Angew Chem., Int. Ed. 2005, 44, 520.

[74] Huo, X.; Yang, G.; Liu, D.; Liu, Y.; Gridnev, I. D.; Zhang, W. Angew. Chem., Int. Ed. 2014, 53, 6776.

[75] Jing, J.; Huo, X.; Shen, J.; Fu, J.; Meng, Q.; Zhang, W. Chem. Commun. 2017, 53, 5151.

[76] Hikawa, H.; Yokoyama, Y. J. Org. Chem. 2011, 76, 8433.

[77] Xie, J.; Xue, S.; Escudero-Adán, E. C.; Kleij, A. W. Angew. Chem., Int. Ed. 2018, 57, 16727.

[78] Xie, J.; Xiao, C.; Belmonte, M. M.; Escudero-Adán, E. C.; Kleij, A. W. ChemSusChem 2019, 12, 3152

[79] Haruki, H.; Yasuda, S.; Nagao, K.; Ohmiya, H. Chem.-Eur. J. 2019,
25,724 .

[80] Zhou, H.; Yang, H.; Yin, H.; Liu, M.; Xia, C.; Jiang, G. RSC Adv. 2014, 4, 25596.

[81] Xie, P.; Wang, J.; Fan, J.; Liu, Y.; Wo, X.; Loh, T.-P. Green Chem. 2017, 19, 2135.

[82] Xie, P.; Wang, J.; Liu, Y.; Fan, J.; Wo, X.; Fu, W.; Sun, Z.; Loh, T.-P. Nat. Commun. 2018, 9, 1321.

[83] Ma, X.; Yu, J.; Zhou, Q.; Yan, R.; Zheng, L.; Wang, L. J. Org. Chem. 2019, 84, 7468

[84] Ma, X.; Yu, J.; Ma, R.; Yan, R.; Zhang, Z. Chin. J. Org. Chem. 2019, 39, 830 (in Chinese) (马献涛，于静，马瑞甜，燕然，张振雷，有机化学，2019，39, 830.)

[85] Blanda, M. T.; Horner, J. H.; Newcomb, M. J. Org. Chem. 1989, $54,4626$.

[86] Cauble, D. F.; Lynch, V.; Krische, M. J. J. Org. Chem. 2003, 68, 15.

[87] Du, L.; Cao, P.; Xing, J.; Lou, Y.; Jiang, L.; Li, L.; Liao, J. Angew. Chem., Int. Ed. 2013, 52, 4207.

[88] Ulatowski, F.; Da browa, K.; Bałakier, T.; Jurczak, J. J. Org. Chem. 2016, 81, 1746.

[89] Thordarson, P. Chem. Soc. Rev. 2011, 40, 1305.

[90] Feng, B.; Li, Y.; Li, H.; Zhang, X.; Xie, H.; Cao, H.; Yu, L.; Xu, Q. J. Org. Chem. 2018, 83, 6769.

[91] Tsuchida, N.; Yamabe, S. J. Phys. Chem. A 2005, 109, 1974.

[92] Lu, C.-J.; Chen, D.-K.; Chen, H.; Wang, H.; Jin, H.; Huang, X.; Gao, J. Org. Biomol. Chem. 2017, 15, 5756

[93] Kumar, D.; Vemula, S. R.; Cook, G. R. Green Chem. 2015, 17, 4300.

[94] Zhou, Q.; Zheng, L.; Ma, B.; Huang, L.; Liu, A.; Cao, X.; Yu, J.; Ma, X. J. Org. Chem. 2020, 85, 5097.

[95] Motokura, K.; Ikeda, M.; Kim, M.; Nakajima, K.; Kawashima, S.; Nambo, M.; Chun, W.-J.; Tanaka, S. ChemCatChem 2018, 10, 4536.

[96] Motokura, K.; Kawashima, S.; Nambo, M.; Manaka, Y.; Chun, W.-J. ChemCatChem 2020, 12, 2783.

(Zhao, C.) 